American Journal of Infectious Diseases 6 (4): 98-102, 2010

ISSN 1553-6203

(C) 2010 Science Publications

\title{
Cerebrospinal Fluid (CSF) Ferritin for Differentiation of Aseptic and Bacterial Meningitis in Adults
}

\author{
${ }^{1}$ Zhinous Bayat Makoo, ${ }^{2}$ Nagmeh Ahadi, ${ }^{3}$ Akbar Hasani, \\ ${ }^{4}$ Roshanak Bayat Makoo and ${ }^{5}$ Omid Mashrabi \\ ${ }^{1}$ Infectious Diseases and Tropical medicine research center, \\ Tabriz University of Medical Sciences, Tabriz, Iran \\ ${ }^{2}$ Infectious Disease Specialist, Faculty of Medicine, \\ Tabriz University of Medical Sciences, Tabriz, Iran \\ ${ }^{3}$ Tabriz University of Medical Sciences, Tabriz, Iran \\ ${ }^{4}$ MSC of Biochemistry, Faculty of Medicine, \\ Islamic Azad university Tabriz branch \\ ${ }^{5}$ General Physician, Faculty of Medicine, \\ Tabriz University of Medical Sciences, Tabriz, Iran
}

\begin{abstract}
Problem statement: Bacterial meningitis is one of the most important causes of mortality and morbidity. Rapid diagnosis of bacterial cause is very important and critical, because early antibiotic therapy prevents complications; different tests have been developed for rapid diagnosis of bacterial meningitis. One of them is CSF ferritin, which is evaluated in this study. Approach: During cross sectional study from 2008-2009 for 2 years, CSF was collects from 61 adults suspected to have meningitis. These patients admitted to Emam Reza and Sina hospitals of Tabriz. CSF was analyzed for ferritin, glucose, protein, cell count, culture and gram stains. Clinical data's were collected for all patients. Collected data's were analyzed by SPSS software. Results: From 61 specimens, 19 cases had bacterial, 19 cases had aseptic meningitis and 23 cases had no criteria for diagnosis of meningitis. CSF ferritin in bacterial meningitis was $231.63 \pm 61.26 \mathrm{ng} \mathrm{dL}^{-1}$ that was significantly higher, than that of aseptic meningitis group. Cut off value for ferritin was estimated $155 \mathrm{ng} \mathrm{dL}^{-1}$ with sensitivity of $94 \%$ and specificity of $21.7 \%$ in this study. Symptoms such as levels of consciousness, fever and seizure were not considered as a criteria for diagnosis of bacterial meningitis $(p>0.05)$. But symptoms such as headache, nausa, vomiting and neck stefness were significantly higher in bacterial and aseptic groups than no meningitis group $(\mathrm{p}<0.05)$. Conclusion: In this study CSF ferritin in bacterial meningitis group was significantly higher than aseptic meningitis group. But due to low specificity of CSF ferritin for differentiation of bacterial from aseptic meningitis, we don't recommend performance of this test in early phase of meningitis course.
\end{abstract}

Key words: CSF ferritin, bacterial meningitis, aseptic meningitis, lactate, Lactate Dehydrogenase (LDH), C-Reactive Protein (CRP), diagnostic criteria, cerebrospinal fluid, antibiotic therapy, regional synthesis, aseptic meningitis, quantitative measuring

\section{INTRODUCTION}

Prior to the advent of antibiotics, bacterial meningitis was considered a fatal disease. Although antibiotic therapy has excelled the prognosis of the patients with bacterial meningitis dramatically, bacterial meningitis is still a considerable cause of mortality and morbidity especially in children (Tunkel and Michael, 2010; Roos and Tyler, 2008; Feigin and Pearlman,
2004). Bacterial meningitis is a life-threatening disease but due to being treatable early diagnosis is of great importance. Differentiating bacterial meningitis from viral meningitis in the acute phase is sometimes baffling for physicians as signs are mostly similar and rapid laboratory tests are not always diagnostic (Roos and Tyler, 2008) as in 10\% of the patients with acute bacterial meningitis, there is cellular count with lymphocytic predominance and even in the beginning

Corresponding Author: Mashrabi Omid, General Physician, Faculty of Medicine, Tabriz University of Medical Sciences, Tabriz, Iran Tel: +98-9144049694 and +98-4226226106 
Am. J. Infect. Dis., 6 (4): 98-102, 2010

of the meningitis process, cellular count and protein levels of cerebrospinal fluid can be in normal range in infants and immunocompromised patients (Tunkel and Michael, 2010; Roos and Tyler, 2008; Tongun et al., 2010). Finally the prognosis of bacterial meningitis mainly depends on the early initiation of appropriate antibiotic therapy (Tunkel and Michael, 2010; Roos and Tyler, 2008).

Different biochemical markers have been used for early diagnosis of bacterial meningitis including Lactate, Lactate Dehydrogenase (LDH), C-Reactive Protein (CRP), ferritin and cytokines (Tunkel and Michael, 2010; Roos and Tyler, 2008). Among these biochemical markers lactate, LDH and CRP have less specificity and measuring cytokines is hardly performed in health Centers. Cerebrospinal fluid ferritin test however is simple and can be performed rapidly. Ferritin molecule has a big spherical structure with a molecular weight of 45000 daltons which cannot penetrate through blood brain barrier considerably and therefore ferritin levels of cerebrospinal fluid are independent from its blood levels. Cerebrospinal fluid ferritin level is almost constant in adults but in children it is a related to the age (Tunkel and Michael, 2010; Roos and Tyler, 2008; Feigin and Pearlman, 2004).

This source of cerebrospinal fluid and meningitis ferritin can be explained by two hypotheses. Considerable increase in cerebrospinal fluid ferritin levels could be due to increase in the permeability, regional synthesis and its being released from inflammatory cells but its moderate increase can also be seen in some neurologic disorders such as cerebrovascular events, leukemic infiltration, dementia, inflammation and infection. Cerebrospinal fluid ferritin levels can also be helpful in diagnosing incompletely treated bacterial meningitis in which culture is negative due to the previous antibiotic therapy (Tunkel and Michael, 2010; Roos and Tyler, 2008).

Considering the importance of early diagnosis in bacterial meningitis and starting primary treatment, this study was carried out to evaluate the diagnostic value of cerebrospinal fluid ferritin in differentiating bacterial meningitis from aseptic meningitis.

\section{MATERIAL AND METHODS}

In a descriptive-analytic study carried out on the patients older than 13 years old with a primary diagnosis of meningitis in the infectious disease wards of Imam Reza and Sina Hospitals from 2008-2009, diagnostic value of cerebrospinal fluid ferritin levels in differentiating bacterial meningitis from aseptic meningitis was evaluated. All patients were performed lumbar puncture through third and fourth lumbar vertebral spaces in decubitus position after the specialist having the site disinfected using alcohol and iodine to assess cerebrospinal fluid.

Patients were divided into three groups based on the specifications of cerebrospinal fluid samples: bacterial meningitis, aseptic meningitis and no meningitis.

The diagnostic criteria of bacterial and aseptic meningitis were presence of more than five leukocytes in cubic millimeter of cerebrospinal fluid in the patients with clinical manifestations of meningitis. The diagnostic criteria of bacterial meningitis were positive culture or positive gram staining of cerebrospinal fluid or dominance of polymorphonuclear cells, low sugar and high protein in cerebrospinal fluid.

Diagnostic criteria for aseptic meningitis were presence of more than five leukocytes in cubic millimeter of cerebrospinal fluid with the dominance of mononuclear cells, almost normal sugar and protein in cerebrospinal fluid and negative gram staining and culture of cerebrospinal fluid.

Cases with less than five leukocytes in cubic millimeter of cerebrospinal fluid were not considered meningitis.

Cerebrospinal fluid ferritin was measured using monoband kits (Accu Bind TM ELISA Microwells) and quantitative measuring using immunoenzmometric approach.

Finally the obtained data from the study were analyzed using SPSS 17 software. Unilateral variance and chi-square tests were used to compare the data. Variables were presented as mean and standard deviation and $\mathrm{p} \leq 0.05$ was considered significant. Cut off point for ferritin and desired sensitivity and specificity for it were extracted from ROC curve.

\section{RESULTS}

61 patients were divided into three groups based on the specifications of cerebrospinal fluid and peripheral white blood cells: group 1 (bacterial meningitis), group 2 (viral meningitis) and group three (no meningitis) (Table 1).

Regarding age, the mean age of the group one, two and three were $39.47 \pm 19.62,43.10 \pm 91.61$ and $57.13 \pm 20.05$ years $(\mathrm{p}=0.018)$ respectively.

Regarding gender no significant difference was observed between three groups $(\mathrm{p}=0.22)$.

Regarding consciousness status $(p=0.07)$, fever $(p=$ $0.43)$, kernig's sign $(\mathrm{p}=0.11)$, Brudzinski sign $(\mathrm{p}=$ $0.11)$ and convulsion $(p=0.16)$ no significant difference was observed between three groups. 
Am. J. Infect. Dis., 6 (4): 98-102, 2010

Table 1: Evaluation of CNS parameters in each three groups

\begin{tabular}{lcccc}
\hline & $\begin{array}{l}\text { Bacterial Meningitis } \\
\text { Mean } \pm \text { Std) }\end{array}$ & $\begin{array}{l}\text { Aseptic Meningitis } \\
(\text { Mean } \pm \text { Std) }\end{array}$ & $\begin{array}{l}\text { Non Meningitis } \\
\text { (Mean } \pm \text { Std) }\end{array}$ & P_Value \\
\hline Leukocyte of serum & $18015.78 \pm 6812.34$ & $11773.68 \pm 3957.81$ & $13000 \pm 5230.07$ & $<0.007$ \\
Leukocyte of CSF & $583.15 \pm 5242.28$ & $204.47 \pm 174.60$ & $2.30 \pm 5.49$ & $<0.001$ \\
Glucose of CSF $\left(\mathrm{mg} \mathrm{d} \mathrm{L}^{-1}\right)$ & $31.94 \pm 24.68$ & $74.73 \pm 17.58$ & $93.39 \pm 53.02$ & $<0.001$ \\
Protein of CSF $\left(\mathrm{mg} \mathrm{dL}^{-1}\right)$ & $139.21 \pm 59.39$ & $49.78 \pm 34.47$ & $32.21 \pm 12.06$ & $<0.001$ \\
LDH of CSF & $161.71 \pm 107.50$ & $39.80 \pm 17.72$ & $34.68 \pm 21.32$ & $<0.001$ \\
Ferritin of CSF $\left(\mathrm{ng} \mathrm{dL}^{-1}\right)$ & $231.63 \pm 61.27$ & $160.31 \pm 41.62$ & $121.21 \pm 45.32$ & $<0.001$ \\
\hline
\end{tabular}

In group 1, 13 people (68.4\%), in group 2, 14 people $(73.7 \%)$ and in group 3, two people $(8.7 \%)$ had headache $(\mathrm{p}=0.001)$.

In group 1,13 people $(68.4 \%)$, in group 2,7 people $(36.8 \%)$ and in group 3, four people (17.4\%) had nausea and vomiting $(\mathrm{p}=0.03)$.

In group 1, 15 people $(78.9 \%)$, in group 2, 10 people $(52.6 \%)$ and in group 3, seven people $(30.4 \%)$ had neck stiffness $(\mathrm{p}=0.007)$.

Blood cultures were positive in one patients (5.3\%) in groups 1 and $3(\mathrm{p}=0.51)$.

Gram staining of cerebrospinal fluid revealed gram-positive cocci in one case $(5.3 \%)$ and grampositive diplococci in one case $(5.3 \%)$.

Cerebrospinal fluid culture aws positive in only four people of the group $1(21.1 \%)(\mathrm{p}=0.009)$.

There was no significant difference between three groups regarding mean peripheral blood sugar $(\mathrm{p}=$ $0.60)$, ESR $(p=0.07)$ and CRP $(p=0.35)$.

CSF ferritin with cutoff point of $168 \mathrm{ng} / \mathrm{ml}$ had sensitivity of $89 \%$ and specificity of $38 \%$, with cutoff point of $125 \mathrm{ng} / \mathrm{ml}$ sensitivity of $78 \%$ and specificity of $34 \%$ and cutoff point of $155 \mathrm{ng} \mathrm{mL}^{-1}$ sensitivity of $94 \%$ and specificity of $21.7 \%$.

\section{DISCUSSION}

At the time being there is no clinical or laboratory diagnostic method which can solely prove or disprove bacterial meningitis instantly and accurately in patients with pleocytosis in cerebrospinal fluid and most methods used for diagnosing bacterial meningitis are associated with serious limitations (Takahashi et al., 1999).

This study was carried out to evaluate cerebrospinal fluid ferritin as a guide in early differentiation of acute bacterial from aseptic meningitis in order to start antibiotic therapy as soon as possible. Therefore patients were studied regarding age, gender and clinical and laboratory signs based on specifications of cerebrospinal fluid in three groups of bacterial meningitis, aseptic meningitis and no meningitis. There was no significant difference between two groups regarding gender distribution. Regarding age, the mean age of the group with no meningitis was significantly higher than the group with bacterial and aseptic meningitis. No significant difference was observed between three groups regarding clinical signs including fever, consciousness status, convulsion, kernig's sign and Brudzinski sign. The difference between groups was significant regarding headache, nausea and vomiting and the neck stiffness however these signs could not be used in differentiating bacterial meningitis from aseptic meningitis due to overlap (Tunkel and Michael, 2010; Roos and Tyler, 2008; Feigin and Pearlman, 2004).

Blood cultures were mostly negative and no significant difference was observed. Gram stating and cerebrospinal fluid culture were reported positive only in few cases of bacterial meningitis. Gram staining of cerebrospinal fluid was reported positive in $60 \%$ to $90 \%$ of the patients with bacterial meningitis (Roos and Tyler, 2008). This difference may be due to incorrect stating techniques and sample reading, antibiotic administration before sampling and delay in sending the samples to the laboratory. There were significant differences in the cerebrospinal fluid analyses of three groups regarding white blood cells count, protein, sugar, lactate dehydrogenase and ferritin. These findings were similar to the findings of KIM (YO et al., 2003).

In this study ferreting cutoff point $168 \mathrm{ng} \mathrm{dL}^{-1}$ (16.8 $\mathrm{ng} \mathrm{mL}^{-1}$ ) was achieved with a sensitivity of $89 \%$ and specificity of $38 \%$ however ferritin cutoff point 155 ng $\mathrm{dL}^{-1}\left(15.5 \mathrm{ng} \mathrm{mL}^{-1}\right)$ had sensitivity of $94 \%$ and specificity of $21.7 \%$. In this study cerebrospinal fluid ferritin was higher in the patients with bacterial meningitis compared to the patients with aseptic meningitis which were similar to the results of a study carried out (Takahashi et al., 1999; YO et al., 2003; Lac et al., 2003; Rahimy et al., 1998).

In two separate studies carried out in Iran by Lak and Rahimipour on 64 patients older than 13 years old and 135 patients younger than 14 years old, cerebrospinal fluid ferritin levels, cutoff point $10 \mathrm{ng}$ $\mathrm{mL}^{-1}$ was considered for the diagnosis of bacterial meningitis and cerebrospinal fluid ferritin compared to protein and sugar was more valuable in diagnosing bacterial meningitis. It was concluded that cerebrospinal fluid ferritin can be used as a rapid test in differentiating bacterial meningitis from aseptic meningitis and febrile convulsive disorder. 
Am. J. Infect. Dis., 6 (4): 98-102, 2010

YO et al. (2003) evaluated the diagnostic power of cerebrospinal fluid ferritin in 203 children with meningitis. Cerebrospinal fluid ferritin was significantly higher in group with bacterial meningitis compared to the groups with viral meningitis and no meningitis and it had a positive relation white blood cell count and cerebrospinal fluid protein but a negative relation with cerebrospinal fluid glucose. Cutoff degree for early diagnosis of bacterial meningitis was $15.6 \mathrm{ng}$ $\mathrm{mL}^{-1}$. Takahashi et al. (1999) also evaluated cerebrospinal fluid ferritin and $\beta 2$ microglobuline in patients with meningitis caused by different etiologies. Cutoff point degree of ferritin was $7.5 \mu \mathrm{g}{ }^{-1} \mathrm{~L}$ in five children with bacterial meningitis which was confirmed by positive culture with a sensitivity of $100 \%$ and specificity of $78 \%$. They concluded that serial measurements of cerebrospinal fluid ferritin are valuable in evaluating response to antibiotic therapy in bacterial meningitis. However their results were applicable on patients older than six months.

Katnik et al. (1995) carried out a study on 47 adults and children with bacterial meningitis confirmed by culture. They reported cerebrospinal fluid ferritin cutoff point $18 \mathrm{ng} \mathrm{mL} \mathrm{mL}^{-1}$ with a positive predictive value of $94.1 \%$ and negative predictive value of $100 \%$. In the study of Milman et al. (1993) ferritin was not helpful in differentiating meningitis from cerebrovascular diseases.

Hospitalized patients in our study were adults with age range of 19-85 years whereas previously mentioned studies have mostly been carried out on children. Ferritin cutoff points obtained in our study were similar to other studies (Katnik, 1995 and Takahashi et al., 1999; YO et al., 2003; Lac et al., 2003; Rahimy et al., 1998). Sensitivity and specificity of ferritin cutoff point was different in our study compared to the other studies (Takahashi et al., 1999; YO et al., 2003; Lac et al., 2003; Rahimy et al., 1998; Katnik, 1995). Low specificity of cerebrospinal fluid ferritin in our study was similar to the study of Sato et al. (1998) and Hallgren et al. (1982).

Sindic, studying ferritin levels in cerebrospinal fluid in the patients with neurologic disorders concluded that cerebrospinal fluid ferritin has low specificity for cerebral infections which is in accordance with our study. Campbell et al. (1986) reported high cerebrospinal fluid ferritin levels in bacterial and fungal meningitis.

Deisenhammer et al. (1997) also reported increase in cerebrospinal fluid ferritin levels as a nonspecific finding in HIV-positive patients with acute neurologic episodes.

\section{CONCLUSION}

In this study, cerebrospinal fluid ferritin levels were significantly higher in the group with bacterial meningitis compared to the group with aseptic meningitis however using ferritin for differentiating bacterial meningitis from aseptic meningitis in the primary stages of the disease is not recommended due to its low specificity.

\section{REFERENCES}

Campbell, D.R., B.S. Skikne and I.D. Cook, 1986. Cerebrospinal fluid ferritin levels in screening for meningism. Arch Neurol., 43: 1257-1260. ISSN: 0003-9942

Deisenhammer, F., R.F. Miller, N.S. Brink and M.J. Harrison, 1997. Cerebrospinal fluid ferritin in HIV infected patients with acute neurological episodes. Genitoutin Med., 73: 181-183. DOI: 10.1136/sti.73.3.181

Feigin, R.D. and E. Pearlman, 2004. Bacterial Meningitis beyond the Neonatal Period. In: Text Book of Pediatric Infectious Disease, Feigin, R.D., G.J. Demmler, J.D. Cherry and S.L. Kaplan, (Eds.). Saunders, Philadelphia, pp: 443-474. ISBN: 10: 0721664482

Hallgren, R., G. Lundqvist, G. Sedin and L. Wide, 1982. Cerebrospinal fluid ferritin in newborn infants. Acute Paediatr Scand, 71: 97-102. DOI: $10.1111 /$ j.1651-2227.1982.tb09378.x

Katnik, R., 1995. A persistent biochemical marker for partially treated meningitis and ventriculitis. J. Child Neurol., 10: 93-99. DOI: 10.1177/088307389501000204

Lac, M., M. Nabavi, S.H. Tagavi and A.A. Kolahy, 2003. Diagnostic capability of ferritin in differentiation of bacterial form viral meningitis. Iranian J. Infect. Dis. Tropical. Med., 8: 42-80.

Milman, N., N.A. Gradual, T.S. Oslen and J.H. Wandall, 1993. Cerebrospinal fluid ferritin in patients with meningitis and cerebral infection or bleeding. Dan. Med. Bull., 40: 490-492. ISSN: 0907-8916

Rahimy, P.A., M.H.R. Fard and A. Jalali, 1998. Diagnostic evaluation of cerebrospinal fluid ferritin in differentiation of bacterial from aseptic meningitis. Med. J. Tab. Uni. Med. SC Persian, 33: 17-24.

Roos, K.L. and K.L. Tyler, 2008. Meningitis. In: Harrison Principle of Internal Medicine, Kasper D.L., E. Braunwald, A.S. Fauci, S.L. Hauser and D.L. Longo et al. (Eds.). MC Graw Hill, New York, pp: 2471-2490. ISBN: 10: 0071402357 
Sato, Y., Y. Honda, T. Asoh, K. Oizumi and Y. Ohshimo et al., 1998. Cerebrospinal fluid ferritin in glioblastoma. J. Neurol., 40: 47-50. DOI: 10.1023/A:1006078521790

Takahashi, S., J. Oki, A. Miyamoto and T. Moriyama, 1999. Beta 2 microglobulin and ferritin in cerebrospinal fluid for evaluation of patients with meningitis of different etiologies. Brain Dev., 21: 192-199. DOI: 10.1016/S0387-7604(99)00017-0

Tongun, T., P. Wannanon, J. W. and W. Phachonpai, 2010. cognitive-enhancing and antioxidant activities of quercetin liposomes in animal model of alzheimer's disease. OnLine J. Biol. Sci., 10: 8491. DOI: 10.3844/ojbsci.2010.84.91
Tunkel, A.R. and S.W. Michael, 2010. Acute Meningitis. In: Principle and Practice of Infectious Disease, Mandell, G.L., R.G. Dollin and J.E. Bennett (Eds.). Churchill Livingstone/Elsevier, Philadelphia, pp: 1083-1119. ISBN: 0443068399

YO, K., J.S. Kang, M.H. Youm and Y.J. Woo, 2003. Diagnostic capability of CSF ferritin in children with meningitis. Pediatric Neurol., 28: 271-276. DOI: $10.1016 / \mathrm{S} 0887-8994(02) 00625-2$ 